\title{
ENERGY SOLUTION TO SCHRÖDINGER-POISSON SYSTEM IN THE TWO-DIMENSIONAL WHOLE SPACE
}

\author{
SATOSHI MASAKI
}

\begin{abstract}
We consider the Cauchy problem of the two-dimensional Schrödinger-Poisson system in the energy class. Though the Newtonian potential diverges at the spatial infinity in the logarithmic order, global well-posedness is proven in both defocusing and focusing cases. The key is a decomposition of the nonlinearity into a sum of the linear logarithmic potential and a good remainder, which enables us to apply the perturbation method. Our argument can be adapted to the one-dimensional problem.
\end{abstract}

\section{INTRODUCTION}

This paper is devoted to the study of the Schödinger-Poisson system

$$
\left\{\begin{array}{l}
i \partial_{t} u+\frac{1}{2} \Delta u=\lambda P u, \quad(t, x) \in \mathbb{R}^{1+2}, \\
-\Delta P=|u|^{2} \\
u(0, x)=u_{0}(x)
\end{array}\right.
$$

where $\lambda$ is a real constant. We suppose $P$ is the Newtonian potential

$$
P=-\frac{1}{2 \pi}\left(\log |x| *|u|^{2}\right)
$$

where $*$ denotes the convolution. For a suitable $u$, this is the unique strong solution of $-\Delta P=|u|^{2}$ under the condition

$$
|\nabla P| \rightarrow 0 \text { as }|x| \rightarrow \infty, \quad \nabla P \in L^{\infty}\left(\mathbb{R}^{2}\right), \quad P(0)=\int_{\mathbb{R}^{2}}(\log |y|)|u(y)|^{2} d y
$$

(see [11). When the dimensions are larger than two, the SchrödingerPoisson system is a special case of the Hartree equation and one of the typical example of the nonlinear Schrödinger equation with a nonlocal nonlinearity, and there is large amount of literature (see [6] and references therein). On the other hand, the two-dimensional case is less studied. In [1, 18, (1.1) is considered with some restrictive assumptions such as a neutrality condition which confirms that the Newtonian potential (1.2) does not diverge at the spatial infinity and in particular belongs to $L^{2}$ space. The Poisson equation is sometimes posed with a background (or doping profile):

$$
-\Delta P=|u|^{2}-b,
$$

where $b$ is a given positive function. Then, the neutrality condition is $\int|u|^{2}-$ $b d x=0$ or equivalently $\mathcal{F}\left(|u|^{2}-b\right)(0)=0$. When we consider the problem in dimensions less than three, this condition is useful to control $P$. Notice that this condition excludes all nontrivial solutions when $b \equiv 0$, and that we need to remove this condition for the study of (1.1). In [11, the above 
assumptions are removed and the existence of a unique local solution is proven for data in the usual Sobolev space $H^{s}\left(\mathbb{R}^{2}\right)(s>2)$ despite the fact that the nonlinear potential diverges at the spatial infinity. Since (1.2) is not necessarily defined for $u \in H^{s}(s>2)$ we introduced a new formula

$$
P=-\frac{1}{2 \pi} \int_{\mathbb{R}^{2}}\left(\log \frac{|x-y|}{|y|}\right)|u(y)|^{2} d y
$$

which makes sense merely if $|u|^{2} \in L^{p}\left(\mathbb{R}^{2}\right)(p \in(1,2))$. We underline that the local solutions given there do not have finite energy (the energy is given in (1.5) below). Our aim in this paper is to prove that there exists a timeglobal solution if initial data has finite energy.

For our analysis, the following reduction is crucial: We guess that the Newtonian potential (1.2) may behave like $-\frac{1}{2 \pi}\|u\|_{L^{2}}^{2} \log |x|$ at the spatial infinity, which will be the bad part of the nonlinearity, and decompose the nonlinearity as

$$
\lambda P u=-\frac{\lambda}{2 \pi}\|u\|_{L^{2}}^{2}(\log \langle x\rangle) u-\frac{\lambda}{2 \pi} u \int_{\mathbb{R}^{2}}\left(\log \frac{|x-y|}{\langle x\rangle}\right)|u(y)|^{2} d y,
$$

where $\langle x\rangle=\left(1+|x|^{2}\right)^{1 / 2}$. We then obtain

$$
i \partial_{t} u+\frac{1}{2} \Delta u+\frac{\lambda}{2 \pi}\|u\|_{L^{2}}^{2}(\log \langle x\rangle) u=-\frac{\lambda}{2 \pi} u \int_{\mathbb{R}^{2}}\left(\log \frac{|x-y|}{\langle x\rangle}\right)|u(y)|^{2} d y .
$$

It will turn out that the bad part of $P$ is correctly extracted from the original nonlinearity and therefore the behavior of the "new nonlinearity" becomes better. Notice that one can also expect that $\|u\|_{L^{2}}$ is conserved because $\lambda$ is a real number. Hence, putting

$$
m:=-\frac{\lambda}{2 \pi}\left\|u_{0}\right\|_{L^{2}}^{2},
$$

we reach to the equation

$$
\left\{\begin{array}{l}
i \partial_{t} u+\left(\frac{1}{2} \Delta-m \log \langle x\rangle\right) u=-\frac{\lambda}{2 \pi} u \int_{\mathbb{R}^{2}}\left(\log \frac{|x-y|}{\langle x\rangle}\right)|u(y)|^{2} d y \\
u(0, x)=u_{0}(x) .
\end{array}\right.
$$

Notice that $-m \log \langle x\rangle$ is now completely independent of $u$ and that it therefore can be regarded as a linear potential. In what follows, we work with this equation. Observe that if there exists a solution to (1.3) conserving $\|u\|_{L^{2}}$, then it is also a solution of (1.1).

Now, the linear part of the equation is not $i \partial_{t}+(1 / 2) \Delta$ but $i \partial_{t}+(1 / 2) \Delta-$ $m \log \langle x\rangle$. Thus, a natural choice of the function space on which we shall work is not the Sobolev space $H^{1}\left(\mathbb{R}^{2}\right)$ any more, but the following one:

$$
\begin{aligned}
& \mathcal{H}:=\left\{u \in H^{1}\left(\mathbb{R}^{2}\right) ; \sqrt{\log \langle x\rangle} u \in L^{2}\right\}, \\
& \|u\|_{\mathcal{H}}:=\|u\|_{H^{1}\left(\mathbb{R}^{2}\right)}+\|\sqrt{\log \langle\cdot\rangle} u\|_{L^{2}\left(\mathbb{R}^{2}\right)} .
\end{aligned}
$$

If $m>0$, that is, if $\lambda<0$, then the above space coincides with the form domain of the positive operator $-\frac{1}{2} \Delta+m \log \langle x\rangle$. Our main result is the following: 
Theorem 1.1. The problem (1.3) is globally well-posed in $\mathcal{H}$. Moreover, the solution conserves $\|u(t)\|_{L^{2}}$ and the energy

$$
E(t)=\frac{1}{2}\|\nabla u(t)\|_{L^{2}}^{2}-\frac{\lambda}{4 \pi} \int_{\mathbb{R}^{2}}(\log |x-y|)|u(t, x)|^{2}|u(t, y)|^{2} d x d y .
$$

Corollary 1.2. The Problem (1.1) is globally well-posed in $\mathcal{H}$.

Remark 1.3. Let $u \in C(\mathbb{R} ; \mathcal{H})$ be a solution of (1.3) (and of (1.1)) given in Theorem 1.1. Then, $v:=u \exp \left(-i \frac{\lambda}{2 \pi} \int_{0}^{t}\|\sqrt{\log |\cdot|} u(s)\|_{L^{2}}^{2} d s\right)$ solves

$$
\left\{\begin{array}{l}
i \partial_{t} v+\frac{1}{2} \Delta v=-\frac{\lambda}{2 \pi} v \int_{\mathbb{R}^{2}}\left(\log \frac{|x-y|}{|y|}\right)|v(y)|^{2} d y, \\
v(0, x)=u_{0}(x) .
\end{array}\right.
$$

Notice that the nonlinearity of (1.6) makes sense without the momentum condition $\sqrt{\log |\cdot|} v \in L^{2}$. This observation explains why existence of a time-local solution can be proven by assuming only $u_{0} \in H^{s}\left(\mathbb{R}^{2}\right)(s>1)$ in [11].

1.1. Consequent results. Our argument is also applicable to (1.1) involving a power type nonlinearity:

$$
\left\{\begin{array}{l}
i \partial_{t} u+\frac{1}{2} \Delta u=\lambda P u+\eta|u|^{p-1} u, \quad(t, x) \in \mathbb{R}^{1+2}, \\
-\Delta P=|u|^{2} \\
u(0, x)=u_{0}(x),
\end{array}\right.
$$

where $\eta$ is a real number and $p \geqslant 2$.

Theorem 1.4. The problem (1.7) is globally well-posed in $\mathcal{H}$ if either one of the following conditions is satisfied:

(1) $\eta \geqslant 0, \lambda \in \mathbb{R}$ and $p \geqslant 2$;

(2) $\eta<0, \lambda \in \mathbb{R}$, and $2 \leqslant p<3$;

(3) $\eta<0, \lambda>0, p=3$, and $\left\|u_{0}\right\|_{\mathcal{H}}$ is small;

(4) $\eta<0, \lambda<0, p \geqslant 3$, and $\left\|u_{0}\right\|_{\mathcal{H}}$ is small.

Moreover, the solution conserves $\|u(t)\|_{L^{2}}$ and the energy

$$
\begin{aligned}
E_{p}(t):= & \frac{1}{2}\|\nabla u(t)\|_{L^{2}}^{2}-\frac{\lambda}{4 \pi} \int_{\mathbb{R}^{2}}(\log |x-y|)|u(t, x)|^{2}|u(t, y)|^{2} d x d y \\
& +\frac{\eta}{p+1}\|u(t)\|_{L^{p+1}}^{p+1} .
\end{aligned}
$$

The proof is done with a straight-forward modification (see Section 4). The case where $p=3$ is known as the $L^{2}$-critical case. Since the $\mathcal{H}$-norm contains derivative, it seems difficult to treat the case $1<p<2$. Nevertheless, we can show global well-posed in a slightly smaller function space $\mathcal{H}^{1,2}:=\left\{u \in H^{1}\left(\mathbb{R}^{2}\right) ; u \log \langle x\rangle \in L^{2}\right\}$.

Theorem 1.5. Suppose $1<p<2$. For $\eta, \lambda \in \mathbb{R}$ The problem (1.7) is globally well-posed in the space $\mathcal{H}^{1,2}$. Moreover, the solution conserves $\|u(t)\|_{L^{2}}$ and the energy $E_{p}(t)$ given in (1.8). 
We can also handle the one-dimensional problem

$$
\left\{\begin{array}{l}
i \partial_{t} u+\frac{1}{2} \partial_{x x} u=-\frac{\lambda}{2}\left(|x| *|u|^{2}\right) u+\eta|u|^{p-1} u, \quad(t, x) \in \mathbb{R}^{1+1}, \\
u(0, x)=u_{0}(x)
\end{array}\right.
$$

where $\lambda, \eta \in \mathbb{R}$ and $p \geqslant 2$. The one dimensional problem was studied in [7, 14, 15]. The global well-posedness of (1.9) was shown in the space $\{f \in$ $\left.H^{1}(\mathbb{R}) ;|x| f \in L^{2}(\mathbb{R})\right\}$ in $[15]$, and in the space $\left\{f \in H^{1}(\mathbb{R}) ; \sqrt{|x|} f \in L^{2}(\mathbb{R})\right\}$ with a presence of background in [7, provided $\lambda>0$ and data is small relative to the background. We can prove the global well-posedness result of (1.9) including these results.

Theorem 1.6. The problem (1.9) is globally well-posed in $\left\{f \in H^{1}(\mathbb{R}) ; \sqrt{|x|} f \in\right.$ $\left.L^{2}(\mathbb{R})\right\}$ if $\lambda \in \mathbb{R}$ and either one of the following conditions is satisfied:

(1) $\eta \geqslant 0, \lambda \in \mathbb{R}$, and $p \geqslant 2$;

(2) $\eta<0, \lambda \in \mathbb{R}$, and $2 \leqslant p<5$;

(3) $\eta<0, \lambda>0, p=5$, and $\left\|u_{0}\right\|_{H^{1}}+\left\|\sqrt{|\cdot|} u_{0}\right\|_{L^{2}}$ is small;

(4) $\eta<0, \lambda<0, p \geqslant 5$, and $\left\|u_{0}\right\|_{H^{1}}+\left\|\sqrt{|\cdot|} u_{0}\right\|_{L^{2}}$ is small.

The solution conserves $\|u\|_{L^{2}}$ and the energy

$\widetilde{E}(t):=\frac{1}{2}\left\|\partial_{x} u\right\|_{L^{2}(\mathbb{R})}^{2}-\frac{\lambda}{2} \iint_{\mathbb{R}^{2}}\left|x-y\left\|\left.u(x)\right|^{2}|u(y)|^{2} d x d y+\frac{\eta}{p+1}\right\| u \|_{L^{p+1}(\mathbb{R})}^{p+1}\right.$.

The one-dimensional version of Theorem 1.5 is as follows, which reproduce the same result in [15, Theorem 2.1] when $\eta<0$ and $\lambda>0$.

Theorem 1.7. Suppose $1<p<2$. For $\eta, \lambda \in \mathbb{R}$ The problem (1.9) is globally well-posed in the space $\Sigma:=\left\{u \in H^{1}\left(\mathbb{R}^{2}\right) ;|x| u \in L^{2}\right\}$. Moreover, the solution conserves $\|u(t)\|_{L^{2}}$ and the energy $\widetilde{E}(t)$ given in (1.10).

As in the two dimensional case, the key is a "reduction" of (1.9) to

$$
\left\{\begin{array}{l}
i \partial_{t} u+\frac{1}{2} \partial_{x x} u+\frac{\lambda\left\|u_{0}\right\|_{L^{2}}^{2}}{2}|x| u=-\frac{\lambda}{2} u \int_{\mathbb{R}}(|x-y|-|x|)|u(y)|^{2} d y+\eta|u|^{p-1} u, \\
u(0, x)=u_{0}(x) .
\end{array}\right.
$$

We briefly mention about other related works. Oh considered in [12] the Cauchy problem of the nonlinear Schrödinger equation with general potential and $L^{2}$-subcritical power-type nonlinearity, and proved global wellposedness in the form domain of $-\frac{1}{2} \Delta+V$, provided the potential $V \geqslant 0$ satisfies $\partial^{\alpha} V \in L^{\infty}$ for $|\alpha| \geqslant 2$ (see also [6]). In particular, the case where the potential $V$ is a quadratic polynomial is extensively studied. In this case, we have several special properties such as explicit representations of linear solutions, called Mehler's formula, and/or of the Heisenberg observables. We refer the reader to [2, 3, 4, 10, 19] for $H^{1}$-subcritical and $H^{1}$-critical powertype nonlinearity and to [5] for $H^{1}$-subcritical Hartree type nonlinearity. In [16], the ground states of (1.1) is treated.

The rest of the paper is organized as follows: We collect some basic estimates in Section 2, and, in Section 3 we prove Theorem 1.1. Section 4 is devoted to the study of (1.7). 


\section{Preliminaries}

2.1. Strichartz estimate. We first summarize the properties on the operator

$$
A:=\frac{1}{2} \Delta-m \log \langle x\rangle,
$$

where $m \neq 0$ is a real constant. For any $m, A$ is essentially self-adjoint on $C_{0}^{\infty}\left(\mathbb{R}^{2}\right)$ (see [13]). Since our potential is sub-quadratic, that is, since $\left|\partial^{\alpha} \log \langle x\rangle\right| \rightarrow 0$ as $|x| \rightarrow \infty$ for $|\alpha|=2$ and $\partial^{\alpha} \log \langle x\rangle \in L^{\infty}$ for $|\alpha| \geqslant 3$, the following estimate is established in [17]: For any $T>0$,

$$
\left\|e^{i t A} \varphi\right\|_{L^{\infty}} \leqslant C|t|^{-1}\|\varphi\|_{L^{1}}
$$

for $t \in[-T, T]$, where $C$ depends on $T$ (see also [8]). Once we know this type of estimate, the Strichartz estimate follows by interpolation. We say that a pair $(q, r)$ is admissible if $2 \leqslant r<\infty$ and $2 / q=\delta(r):=1-2 / r$.

Lemma 2.1 (Strichartz's estimate). For any $T>0$, the following properties hold:

- Suppose $\varphi \in L^{2}\left(\mathbb{R}^{2}\right)$. For any admissible pair $(q, r)$, there exists a constant $C=C(T, q, r)$ such that

$$
\left\|e^{i t A} \varphi\right\|_{L^{q}\left((-T, T) ; L^{r}\right)} \leqslant C\|\varphi\|_{L^{2}} .
$$

- Let $I \subset(-T, T)$ be an interval and $t_{0} \in \bar{I}$. For any admissible pairs $(q, r)$ and $(\gamma, \rho)$, there exists a constant $C=C(t, q, r, \gamma, \rho)$ such that

$$
\left\|\int_{t_{0}}^{t} e^{i(t-s) A} F(s) d s\right\|_{L^{q}\left(I ; L^{r}\right)} \leqslant C\|F\|_{L^{\gamma^{\prime}\left(I ; L^{\rho^{\prime}}\right)}}
$$

for every $F \in L^{\gamma^{\prime}}\left(I ; L^{\rho^{\prime}}\right)$.

\subsection{Some estiamtes.}

Lemma 2.2. Let $W$ be an arbitrary weight function such that $\nabla W, \Delta W \in$ $L^{\infty}\left(\mathbb{R}^{2}\right)$. It holds for all $T>0$, admissible pair $(q, r)$, and $\varphi \in \mathcal{H}$ that

$$
\begin{aligned}
& \left\|\left[\nabla, e^{i t A}\right] \varphi\right\|_{L^{q}\left((-T, T) ; L^{r}\right)} \leqslant C|T|\|\varphi\|_{2}, \\
& \left\|\left[W, e^{i t A}\right] \varphi\right\|_{L^{q}\left((-T, T) ; L^{r}\right)} \leqslant C|T|\|(1+\nabla) \varphi\|_{2} .
\end{aligned}
$$

Proof. Since $v=e^{i t A} \varphi$ solves $i \partial_{t} v+A v=0$, an explicit calculation shows

$$
\left[\nabla, e^{i t A}\right] \varphi=-i \int_{0}^{t} e^{i(t-s) A} \frac{m x}{1+x^{2}} e^{i s A} \varphi d s
$$

and

$$
\left[W, e^{i t A}\right] \varphi=i \int_{0}^{t} e^{i(t-s) A}\left(\nabla W \cdot \nabla+\frac{1}{2} \Delta W\right) e^{i s A} \varphi d s .
$$

The Strichartz estimate therefore gives the desired estimates.

The following is useful for estimates of the nonlinearity in (1.3). 
Lemma 2.3. Set a function

$$
K(x, y)=\frac{\log \frac{|x-y|}{\langle x\rangle}}{1+\log \langle y\rangle}
$$

of $x, y \in \mathbb{R}^{2}$. For any $p \in[1, \infty)$ and $\varepsilon>0$, there exist a function $W(x, y) \geqslant$ 0 with $\|W\|_{L_{y}^{\infty} L_{x}^{p}} \leqslant \varepsilon$ and a constant $C_{0}$ such that

$$
|K(x, y)| \leqslant C_{0}+W(x, y)
$$

holds for all $(x, y) \in \mathbb{R}^{2+2}$.

Proof. Take $\eta \in(0,1]$ and set $W(x, y)=|K(x, y)| \mathbf{1}_{|x-y| \leqslant \eta}(x, y)$. If $\eta$ is sufficiently small then

$$
\|W(\cdot, y)\|_{L^{p}} \leqslant \frac{\|\log |x|\|_{L^{p}(|x| \leqslant \eta)}+\log \langle|y|+\eta\rangle\|1\|_{L^{p}(|x| \leqslant \eta)}}{1+\log \langle y\rangle} \leqslant \varepsilon
$$

since $\log |x|$ belongs to $L_{\text {loc }}^{p}\left(\mathbb{R}^{2}\right)$ for all $p<\infty$. Moreover, by (2.12) of [11,

$$
\sup _{|x-y| \geqslant \eta} K(x, y) \leqslant 1+\log \frac{\sqrt{3}}{\eta}
$$

for any $\eta \leqslant 1$, which completes the proof.

Remark 2.4. In 1D case, the corresponding estimate is

$$
\left\|\frac{|x-y|-|x|}{1+|y|}\right\|_{L_{x, y}^{\infty}\left(\mathbb{R}^{2}\right)} \leqslant 1 .
$$

\section{Proof of the theorem}

\subsection{Local well-posedness.}

Lemma 3.1. Let $\left(q_{0}, r_{0}\right)$ be an admissible pair with $r_{0}>2$. For any $u_{0} \in \mathcal{H}$, there exist an existence time $T=T\left(\left\|u_{0}\right\|_{\mathcal{H}}\right)$ and a unique solution $u \in$ $C((-T, T) ; \mathcal{H}) \cap L^{q_{0}}\left((-T, T) ; L^{r_{0}}\right) \cap C^{1}\left((-T, T) ; \mathcal{H}^{*}\right)$. The solution conserves $\|u(t)\|_{L^{2}}$ and the energy (1.5). Moreover, the map $u_{0} \mapsto u$ is continuous from $\mathcal{H}$ to $C((-T, T) ; \mathcal{H})$.

Proof. We write $L^{p}((-T, T) ; X)=L_{T}^{p} X$, for short. Define a Banach space

$$
\mathcal{H}_{T, M}:=\left\{f \in L^{\infty}((-T, T) ; \mathcal{H}) ;\|f\|_{\mathcal{H}_{T}} \leqslant M\right\}
$$

with norm

$$
\|f\|_{\mathcal{H}_{T}}:=\|f\|_{L_{T}^{\infty} \mathcal{H}}+\|f\|_{L_{T}^{q_{0}} W^{1, r_{0}}}+\|\sqrt{\log \langle x\rangle} f\|_{L_{T}^{q_{0}} L^{r_{0}}} .
$$

We show that if $r_{0}>2$ then there exist $M=M\left(\left\|u_{0}\right\|_{\mathcal{H}}\right)$ and $T=T\left(\left\|u_{0}\right\|_{\mathcal{H}}\right)$ such that

$$
\begin{aligned}
Q[u](t, x): & =\left(e^{i t A} u_{0}\right)(x) \\
& +\frac{i}{2 \pi}\left(\int_{0}^{t} e^{i(t-s) A}\left(\int_{\mathbb{R}^{2}} \log \frac{|\cdot-y|}{\langle\cdot\rangle}|u(s, y)|^{2} d y\right) u(s, \cdot) d s\right)(x)
\end{aligned}
$$

becomes a contraction map from $\mathcal{H}_{T, M}$ to itself, where $A$ is defined in (2.1). 
Set

$$
K(x, y)=\frac{\log \frac{|x-y|}{\langle x\rangle}}{1+\log \langle y\rangle} .
$$

Then, by Lemma 2.3, there exist a nonnegative function $W \in L_{y}^{\infty} L_{x}^{r_{0}^{\prime}}$ and a constant $C_{0}$ such that

$$
|K(x, y)| \leqslant C_{0}+W(x, y) .
$$

Recall that $r_{0} \in(2, \infty)$ and so $r_{0}^{\prime}:=r_{0} /\left(r_{0}-1\right) \in(1,2)$. We hence see that

$$
P u=\iint K(x, y)(1+\log \langle y\rangle)|u(y)|^{2} u(x) d y d x
$$

satisfies

$$
\|P u\|_{L^{2}} \leqslant C\left(\|u\|_{L^{2}}+\|u\|_{L^{r_{0}}}\right)\|\sqrt{1+\log \langle x\rangle} u\|_{L^{2}}^{2} .
$$

Take $L_{T}^{1}$ norm to yield

$$
\|P u\|_{L_{T}^{1} L^{2}} \leqslant C\left(T\|u\|_{L_{T}^{\infty} L^{2}}+T^{\frac{1}{2}+\frac{1}{r_{0}}}\|u\|_{L_{T}^{q_{0}} L^{r_{0}}}\right)\|\sqrt{1+\log \langle x\rangle} u\|_{L_{T}^{\infty} L^{2}}^{2} .
$$

By the Strichartz estimate, we end up with

$$
\|Q[u]\|_{L_{T}^{\infty} L^{2}}+\|Q[u]\|_{L_{T}^{q_{0}} L^{r_{0}}} \leqslant C\left\|u_{0}\right\|_{L^{2}}+C\left(T+T^{\frac{1}{2}+\frac{1}{r_{0}}}\right)\|u\|_{\mathcal{H}_{T}}^{3} .
$$

We next estimate $\nabla Q[u]$. One easily sees that

$$
\begin{aligned}
\nabla Q[u]= & e^{i t A} \nabla u_{0}-i \int_{0}^{t} e^{i(t-s) A} \nabla(P u)(s) d s \\
& +\left[\nabla, e^{i t A}\right] u_{0}-i \int_{0}^{t}\left[\nabla, e^{i(t-s) A}\right](P u)(s) d s .
\end{aligned}
$$

We deduce from Lemma 2.2 with $(q, r)=(\infty, 2)$ that

$$
\int_{0}^{t}\left\|\left[\nabla, e^{i(t-s) A}\right](P u)(s)\right\|_{L^{2}} d s \leqslant \int_{0}^{t}(t-s)\|P u(s)\|_{L^{2}} d s \leqslant|t|\|P u\|_{L_{T}^{1} L^{2}} .
$$

The right hand side is bounded as in (3.1). $\left[\nabla, e^{i t A}\right] u_{0}$ is handled similarly. Mimicking (3.1), we infer that

$$
\|P \nabla u\|_{L_{T}^{1} L^{2}} \leqslant C\left(T\|\nabla u\|_{L_{T}^{\infty} L^{2}}+T^{\frac{1}{2}+\frac{1}{r_{0}}}\|\nabla u\|_{L_{T}^{q_{0}} L^{r_{0}}}\right)\|\sqrt{1+\log \langle x\rangle} u\|_{L_{T}^{\infty} L^{2}}^{2}
$$

Now, let us estimate $(\nabla P) u$. It writes

$$
(\nabla P)(x) u(x)=\left(\int_{\mathbb{R}^{2}}\left(\frac{x-y}{|x-y|^{2}}-\frac{x}{1+x^{2}}\right)|u(y)|^{2} d y\right) u(x),
$$

and so

$$
\begin{aligned}
\|(\nabla P) u\|_{L^{2}} & \leqslant C\left\|\left(|x|^{-1} *|u|^{2}\right)+\langle\cdot\rangle^{-1}\right\| u\left\|_{L^{2}}^{2}\right\|_{L^{\frac{2 r_{0}}{r_{0}-2}}}\|u\|_{L^{r_{0}}} \\
& \leqslant C\left(\|u\|_{L^{\frac{2 r_{0}}{r_{0}-1}}}^{2}+\|u\|_{L^{2}}^{2}\right)\|u\|_{L^{r_{0}}} \\
& \leqslant C\left(\|u\|_{L^{2}}^{2}+\|\nabla u\|_{L^{2}}^{2}\right)\|u\|_{L^{r_{0}}}
\end{aligned}
$$


by the Hardy-Littlewood-Sobolev and the Sobolev inequalities. We see that

$$
\|(\nabla P) u\|_{L_{T}^{1} L^{2}} \leqslant C\left(T\|u\|_{L_{T}^{\infty} L^{2}}^{2}+T^{\frac{1}{2}+\frac{1}{r_{0}}}\|u\|_{L_{T}^{q_{0}} L^{r_{0}}}^{2}\right)\|u\|_{L_{T}^{\infty} L^{2}} .
$$

We deduce from the Strichartz estimate that

$$
\|\nabla Q[u]\|_{L_{T}^{\infty} L^{2}}+\|\nabla Q[u]\|_{L_{T}^{q_{0}} L^{r_{0}}} \leqslant C\left\|\nabla u_{0}\right\|_{\mathcal{H}}+C\left(T+T^{\frac{1}{2}+\frac{1}{r_{0}}}\right)\|u\|_{\mathcal{H}_{T}}^{3} .
$$

Let us proceed to the estimate of $\sqrt{\log \langle x\rangle} Q[u]$. It holds that

$$
\begin{aligned}
\sqrt{1+\log \langle x\rangle} Q[u]= & e^{i t A} \sqrt{1+\log \langle x\rangle} u_{0}-i \int_{0}^{t} e^{i(t-s) A} \sqrt{1+\log \langle x\rangle} P u(s) d s \\
& +R
\end{aligned}
$$

where

$$
R=\left[\sqrt{1+\log \langle x\rangle}, e^{i t A}\right] u_{0}-i \int_{0}^{t}\left[\sqrt{1+\log \langle x\rangle}, e^{i(t-s) A}\right] P u(s) d s .
$$

A use of Lemma 2.2 with $W=\sqrt{1+\log \langle x\rangle}$ yields

$$
\begin{aligned}
\|R\|_{L_{T}^{\infty} L^{2}}+\|R\|_{L_{T}^{q_{0}} L^{r_{0}}} & \leqslant C T\left\|u_{0}\right\|_{\mathcal{H}}+C T\|(1+\nabla)(P u)\|_{L_{T}^{1} L^{2}} \\
& \leqslant C T\left\|u_{0}\right\|_{\mathcal{H}}+C T\left(T+T^{\frac{1}{2}+\frac{1}{r_{0}}}\right)\|u\|_{\mathcal{H}_{T}}^{3}
\end{aligned}
$$

where we have used (3.1), (3.3), and (3.4). As in (3.1), it holds that

$$
\begin{aligned}
\|P(W u)\|_{L_{T}^{1} L^{2}} & \leqslant C\left(T\|W u\|_{L_{T}^{\infty} L^{2}}+T^{\frac{1}{2}+\frac{1}{r_{0}}}\|W u\|_{L_{T}^{q_{0}} L^{r_{0}}}\right)\|W u\|_{L_{T}^{\infty} L^{2}}^{2} \\
& \leqslant C\left(T+T^{\frac{1}{2}+\frac{1}{r_{0}}}\right)\|u\|_{\mathcal{H}_{T}}^{3},
\end{aligned}
$$

where $W=\sqrt{1+\log \langle x\rangle}$. We conclude from the Strichartz estimate, (3.2), and (3.5) that

$$
\|Q[u]\|_{\mathcal{H}_{T}} \leqslant C_{1}\left\|u_{0}\right\|_{\mathcal{H}}+C_{2}\left(T+T^{\frac{1}{2}+\frac{1}{r_{0}}}\right)\|u\|_{\mathcal{H}_{T}}^{3} .
$$

A similar argument shows

$$
\left\|Q\left[u_{1}\right]-Q\left[u_{2}\right]\right\|_{\mathcal{H}_{T}} \leqslant C_{3}\left(T+T^{\frac{1}{2}+\frac{1}{r_{0}}}\right)\left(\left\|u_{1}\right\|_{\mathcal{H}_{T}}+\left\|u_{2}\right\|_{\mathcal{H}_{T}}\right)^{2}\left\|u_{1}-u_{2}\right\|_{\mathcal{H}_{T}} .
$$

Thus, if we take $M \geqslant 2 C_{1}\left\|u_{0}\right\|_{\mathcal{H}}$ then there exists $T=T(M)$ such that $Q$ is a contraction map from $\mathcal{H}_{T, M}$ to itself.

The conservations of $\|u(t)\|_{L^{2}}$ is shown by multiplying (1.3) by $\bar{u}$ and integrating the imaginary part. To prove the energy conservation, we need a regularizing argument. Note that (1.3) can be solved also in the space $\left\{f \in H^{2}\left(\mathbb{R}^{2}\right): \log \langle x\rangle f \in L^{2}\right\}$, which is one of dense subsets of $\mathcal{H}$, in an essentially same way. We omit details.

3.2. Global existence. We first give a useful blow-up criteria.

Lemma 3.2. Suppose $u_{0} \in \mathcal{H}$. Let $u \in C\left(\left(-T_{\min }, T_{\max }\right) ; \mathcal{H}\right)$ be a unique maximal solution given by Lemma 3.1. If $T_{\max }<\infty$ (resp. $\left.T_{\min }<\infty\right)$, then $\|\nabla u(t)\|_{L^{2}} \rightarrow \infty$ as $t \uparrow T_{\max }$ (resp. $\left.t \downarrow-T_{\min }\right)$. 
Proof. We only consider positive time. Suppose $T_{\max }<\infty$. Then, $\|u(t)\|_{\mathcal{H}}$ has to diverge as $t \uparrow T_{\max }$. Otherwise, we can extend the solution beyond $T_{\max }$ by Lemma 3.1. Recall that $\|u(t)\|_{L^{2}}=\left\|u_{0}\right\|_{L^{2}}$. Since

$$
\begin{aligned}
\frac{d}{d t}\|\sqrt{\log \langle x\rangle} u(t)\|_{L^{2}}^{2} & =2 \operatorname{Re} \int(\log \langle x\rangle) \partial_{t} u(t) \overline{u(t)} d x \\
& =-\operatorname{Im} \int(\log \langle x\rangle) \Delta u(t) \overline{u(t)} d x \\
& =\operatorname{Im} \int \frac{x}{1+x^{2}} \cdot \nabla u(t) \overline{u(t)} d x
\end{aligned}
$$

it holds that

$\left\|\sqrt{\log \langle x\rangle} u\left(t_{2}\right)\right\|_{L^{2}}^{2} \leqslant\left\|\sqrt{\log \langle x\rangle} u\left(t_{1}\right)\right\|_{L^{2}}^{2}+\left|t_{2}-t_{1}\right|\|\nabla u\|_{L^{\infty}\left(\left(t_{1}, t_{2}\right) ; L^{2}\right)}\left\|u_{0}\right\|_{L^{2}}$ for all $-T_{\min }<t_{1}<t_{2}<T_{\max }$. This implies that if we assume

$$
\limsup _{t \uparrow T_{\max }}\|\nabla u(t)\|_{L^{2}}<\infty
$$

then $\|u(t)\|_{\mathcal{H}}$ never blows up. We hence obtain the lemma.

Remark 3.3. As in [9], the solution breaks down with concentration at a point if $\|\sqrt{\log \langle x\rangle} u(t)\|_{L^{2}}=0$. However, this does not occur when $\|\nabla u(t)\|$ is bounded above. Indeed, since

$$
\|u\|_{L^{2}(|x|<r)} \leqslant\|1\|_{L^{4}(|x|<r)}\|u\|_{L^{4}} \leqslant C r^{\frac{1}{2}}\|\nabla u\|_{L^{2}}^{\frac{1}{2}}
$$

for any $r>0$ and since

$$
\|u\|_{L^{2}(|x|<r)}=\left\|u_{0}\right\|_{L^{2}}-\|u\|_{L^{2}(|x| \geqslant r)} \geqslant\left\|u_{0}\right\|_{L^{2}}-\frac{\|\sqrt{\log \langle x\rangle} u\|_{L^{2}}}{(\log \langle r\rangle)^{1 / 2}}
$$

by letting $r=\|\sqrt{\log \langle x\rangle} u\|_{L^{2}}$, we obtain

$$
\|\sqrt{\log \langle x\rangle} u\|_{L^{2}}^{-\frac{1}{2}} \leqslant C\left(\frac{\|\sqrt{\log \langle x\rangle} u\|_{L^{2}}}{\log \left\langle\|\sqrt{\log \langle x\rangle} u\|_{L^{2}}\right\rangle}\right)^{\frac{1}{2}}+C\|\nabla u\|_{L^{2}}^{\frac{1}{2}},
$$

which implies $\|\sqrt{\log \langle x\rangle} u\|_{L^{2}}$ is strictly positive if $\|\nabla u\|_{L^{2}}<\infty$.

Proof of Theorem 1.1. Let us establish a priori estimate of $\|\nabla u(t)\|_{L^{2}}$.

We first consider the case $\lambda<0$. Since $\log |x| \geqslant 0$ for $|x| \geqslant 1$,

$$
\begin{aligned}
& -\frac{\lambda}{4 \pi} \iint_{\mathbb{R}^{2+2}} \log |x-y||u(x)|^{2}|u(y)|^{2} d x d y \\
& \geqslant-\frac{|\lambda|}{4 \pi} \iint_{|x-y|<1}|\log | x-y|||u(x)|^{2}|u(y)|^{2} d x d y \\
& \geqslant-\frac{|\lambda|}{4 \pi}\|\log |x|\|_{L^{2}(|x| \leqslant 1)}^{2}\|u\|_{L^{4}}^{2}\|u\|_{L^{2}}^{2}
\end{aligned}
$$

By the $L^{2}$-conservation and the Sobolev embedding, we have

$$
\|\nabla u(t)\|_{L^{2}}^{2} \leqslant 2 E_{0}+C\|\nabla u(t)\|_{L^{2}} .
$$

Therefore, there exists a constant $M$ independent of $t$ such that $\|\nabla u(t)\|_{L^{2}} \leqslant$ $M$. 
We now suppose $\lambda>0$. By Lemma 2.3, for any $\varepsilon>0$ there exists a constant $C_{0}$ such that the following estimate holds:

$$
\begin{aligned}
& \frac{\lambda}{4 \pi} \iint_{\mathbb{R}^{2+2}} \log |x-y||u(x)|^{2}|u(y)|^{2} d x d y \\
& \leqslant \frac{\lambda}{4 \pi} \iint_{\mathbb{R}^{2+2}} \log \frac{|x-y|}{\langle x\rangle}|u(x)|^{2}|u(y)|^{2} d x d y+\frac{\lambda}{4 \pi}\left\|u_{0}\right\|_{L^{2}}^{2}\|\sqrt{\log \langle x\rangle} u\|_{L^{2}}^{2} \\
& \leqslant \frac{\lambda}{4 \pi}\left(C_{0}\left\|u_{0}\right\|_{L^{2}}^{2}+\varepsilon\|u\|_{L^{4}}^{2}\right)\|\sqrt{1+\log \langle x\rangle} u\|_{L^{2}}^{2}+\frac{\lambda}{4 \pi}\left\|u_{0}\right\|_{L^{2}}^{2}\|\sqrt{\log \langle x\rangle} u\|_{L^{2}}^{2} \\
& \leqslant \frac{\lambda C_{0}}{4 \pi}\left\|u_{0}\right\|_{L^{2}}^{4}+\frac{\lambda\left(C_{0}+1\right)}{4 \pi}\left\|u_{0}\right\|_{L^{2}}^{2}\|\sqrt{\log \langle x\rangle} u\|_{L^{2}}^{2}+C \varepsilon\left\|u_{0}\right\|_{L^{2}}^{3}\|\nabla u\|_{L^{2}} \\
& \quad+C \varepsilon\left\|u_{0}\right\|_{L^{2}}\|\nabla u\|_{L^{2}}\|\sqrt{\log \langle x\rangle} u\|_{L^{2}}^{2} \\
& \leqslant C_{1}+C_{2}(\varepsilon+|t|) \sup _{s \in[0, t]}\|\nabla u(s)\|_{L^{2}}+C_{3} \varepsilon|t| \sup _{s \in[0, t]}\|\nabla u(s)\|_{L^{2}}^{2},
\end{aligned}
$$

where $C_{i}(i=1,2,3)$ depends only on $\lambda, C_{0},\left\|u_{0}\right\|_{\mathcal{H}}$, and $\varepsilon$. Fix $T>0$. Taking $\varepsilon<\left(8 C_{3} T\right)^{-1}$, we deduce from the conservation of $E(t)$ that

$$
\left(\sup _{s \in[0, t]}\|\nabla u(s)\|_{L^{2}}\right)^{2} \leqslant 4 E(0)+4 C_{1}+4 C_{2}(\varepsilon+2 T) \sup _{s \in[0, t]}\|\nabla u(s)\|_{L^{2}}
$$

for $0 \leqslant t \leqslant 2 T$. This implies that

$$
\sup _{t \in[0,2 T]}\|\nabla u(t)\|_{L^{2}} \leqslant C\left(\left\|u_{0}\right\|_{\mathcal{H}}, T\right)<\infty .
$$

Since $T$ is arbitrary, we obtain the global existence.

\section{REMARKS ON THE PROBLEM WITH POWER NONLINEARITY}

We give a rough sketch of the proofs of Theorem 1.4 and 1.5 in this section.

Proof of Theorem 1.4. The local well-posedness part holds if $p \geqslant 2$ as in the proof of Lemma 3.1. The restriction $p \geqslant 2$ is required when we estimate

$$
\begin{gathered}
\left|\nabla\left(\left|u_{1}\right|^{p-1} u_{1}-\left|u_{2}\right|^{p-1} u_{2}\right)\right| \leqslant C_{p}\left(\left|u_{1}\right|^{p-2}+\left|u_{2}\right|^{p-2}\right)\left(\left|\nabla u_{1}\right|+\left|\nabla u_{2}\right|\right)\left|u_{1}-u_{2}\right| \\
+C_{p}\left(\left|u_{1}\right|^{p-1}+\left|u_{2}\right|^{p-1}\right)\left|\nabla\left(u_{1}-u_{2}\right)\right| .
\end{gathered}
$$

By exactly the same argument as in Lemma 3.2, the problem of global existence boils down to obtaining an a priori bound of $\|\nabla u(t)\|_{L^{2}}$. Recall that the conserved energy is

$$
\begin{aligned}
E_{p}(t):= & \frac{1}{2}\|\nabla u(t)\|_{L^{2}}^{2}-\frac{\lambda}{4 \pi} \int_{\mathbb{R}^{2}}(\log |x-y|)|u(t, x)|^{2}|u(t, y)|^{2} d x d y \\
& +\frac{\eta}{p+1}\|u(t)\|_{L^{p+1}}^{p+1} .
\end{aligned}
$$

The case $\eta>0$. We have

$$
\|\nabla u(t)\|_{L^{2}}^{2} \leqslant E_{p}(t)+\frac{\lambda}{4 \pi} \int_{\mathbb{R}^{2}}(\log |x-y|)|u(t, x)|^{2}|u(t, y)|^{2} d x d y .
$$

By the same argument as in the case $\eta=0$, we prove global existence. 
The case $\eta<0$ and $\lambda<0$. Since

$$
\frac{|\eta|}{p+1}\|u(t)\|_{L^{p+1}}^{p+1} \leqslant C_{\eta, p}\left\|u_{0}\right\|_{L^{2}}^{2}\|\nabla u(t)\|_{L^{2}}^{p-1},
$$

we obtain

$$
\|\nabla u(t)\|_{L^{2}}^{2} \leqslant 2 E_{0}+C\|\nabla u(t)\|_{L^{2}}+2 C_{\eta, p}\left\|u_{0}\right\|_{L^{2}}^{2}\|\nabla u(t)\|_{L^{2}}^{p-1}
$$

as in (3.6). Uniform bound of $\|\nabla u(t)\|_{L^{2}}$ is then obtained either the case $p<3$ or the case $p \geqslant 3$ and $\left\|u_{0}\right\|_{L^{2}}$ is small.

The case $\eta<0$ and $\lambda>0$. As in (3.7), for any $T>0$, there exist $\varepsilon, C_{1}$, and $C_{2}$ such that

$$
\begin{aligned}
\left(\sup _{s \in[0, t]}\|\nabla u(s)\|_{L^{2}}\right)^{2} \leqslant & 4 E(0)+4 C_{1}+4 C_{2}(\varepsilon+2 T) \sup _{s \in[0, t]}\|\nabla u(s)\|_{L^{2}} \\
& +4 C_{\eta, p}\left\|u_{0}\right\|_{L^{2}}^{2}\left(\sup _{s \in[0, t]}\|\nabla u(s)\|_{L^{2}}\right)^{p-1} .
\end{aligned}
$$

for $t \leqslant 2 T$. Therefore, if $p<3$ or if $p=3$ and $\left\|u_{0}\right\|_{L^{2}}$ is small, we obtain

$$
\sup _{t \in[0,2 T]}\|\nabla u(t)\|_{L^{2}} \leqslant C\left(\left\|u_{0}\right\|_{\mathcal{H}}, T\right)<\infty .
$$

This concludes the proof of Theorem 1.4,

Proof of Theorem 1.5. We denote $L^{p}((-T, T) ; X)=L_{T}^{p} X$. Our strategy for local well-posedness is to use the contraction argument in a complete metric space $\left(\mathcal{H}_{T, M}^{1,2}, d\right)$, where

$$
\begin{aligned}
& \mathcal{H}_{T, M}^{1,2}:=\left\{f \in C\left((-T, T) ; H^{1}\right) ;\|f\|_{\mathcal{H}_{T}^{1,2}} \leqslant M\right\}, \\
& \|f\|_{\mathcal{H}_{T}^{1,2}}:=\|f\|_{L_{T}^{\infty} \mathcal{H}}+\|f\|_{L_{T}^{q_{0}} W^{1, r_{0}}}+\|f \log \langle x\rangle\|_{L_{T}^{q_{0}} L^{r_{0}}}
\end{aligned}
$$

for an admissible pair $\left(q_{0}, r_{0}\right)$ with $r_{0}>2$, and the metric $d$ is given by

$$
d(f, g)=\|f-g\|_{L_{T}^{\infty} L^{2}}+\|f-g\|_{L_{T}^{q_{0} L^{r_{0}}}} .
$$

We shall show

$$
\begin{aligned}
Q[u](t, x):=\left(e^{i t A} u_{0}\right)(x) & \\
+\frac{i}{2 \pi}\left(\int_{0}^{t} e^{i(t-s) A}\left(\int_{\mathbb{R}^{2}} \log \frac{|\cdot-y|}{\langle\cdot\rangle}|u(s, y)|^{2} d y\right) u(s, \cdot) d s\right)(x) & \quad-i \eta\left(\int_{0}^{t} e^{i(t-s) A}\left(|u|^{p-1} u\right)(s) d s\right)(x)
\end{aligned}
$$

is a contraction map in $\left(\mathcal{H}_{T, M}^{1,2}, d\right)$. Mimicking the proof of Lemma 3.1, one shows that for any $M>0$, there exists $T>0$ such that $Q: \mathcal{H}_{T, M}^{1,2} \rightarrow \mathcal{H}_{T, M}^{1,2}$. To prove $Q$ is a contraction with respect to the metric $d$, the following 
estimate is crucial:

$$
\begin{aligned}
& \left\|\left(\int_{\mathbb{R}^{2}} \log \frac{|x-y|}{\langle x\rangle}\left|u_{1}(y)\right|^{2} d y\right) u_{1}-\left(\int_{\mathbb{R}^{2}} \log \frac{|x-y|}{\langle x\rangle}\left|u_{2}(y)\right|^{2} d y\right) u_{2}\right\|_{L^{2}} \\
& \leqslant\left\|\left(\int_{\mathbb{R}^{2}} \log \frac{|x-y|}{\langle x\rangle}\left|u_{1}(y)\right|^{2} d y\right)\left(u_{1}-u_{2}\right)\right\|_{L^{2}} \\
& \quad+\left\|\left(\int_{\mathbb{R}^{2}} \log \frac{|x-y|}{\langle x\rangle}\left(\left|u_{1}(y)\right|^{2}-\left|u_{2}(y)\right|^{2}\right) d y\right) u_{2}\right\|_{L^{2}} \\
& \leqslant \\
& \quad C\left(\left\|u_{1}\right\|_{L^{2}}^{2}+\left\|\sqrt{\log \langle x\rangle} u_{1}\right\|_{L^{2}}^{2}\right)\left(\left\|u_{1}-u_{2}\right\|_{L^{2}}+\left\|u_{1}-u_{2}\right\|_{L^{r_{0}}}\right) \\
& \quad+C\left(\left\|\left|u_{1}\right|^{2}-\left|u_{2}\right|^{2}\right\|_{L^{1}}+\left\|\left(\left|u_{1}\right|^{2}-\left|u_{2}\right|^{2}\right) \log \langle x\rangle\right\|_{L^{1}}\right)\left(\left\|u_{2}\right\|_{L^{2}}+\left\|u_{2}\right\|_{L^{r_{0}}}\right) \\
& \leqslant \\
& \quad C\left(\left\|u_{1}\right\|_{L^{2}}^{2}+\left\|\sqrt{\log \langle x\rangle} u_{1}\right\|_{L^{2}}^{2}\left(\left\|u_{1}-u_{2}\right\|_{L^{2}}+\left\|u_{1}-u_{2}\right\|_{L^{r_{0}}}\right)\right. \\
& \quad+C\left(\left\|u_{1}\right\|_{L^{2}}+\left\|u_{2}\right\|_{L^{2}}+\left\|u_{1} \log \langle x\rangle\right\|_{L^{2}}+\left\|u_{2} \log \langle x\rangle\right\|_{L^{2}}\right) \\
& \quad \times\left(\left\|u_{2}\right\|_{L^{2}}+\left\|u_{2}\right\|_{L^{r_{0}}}\right)\left\|u_{1}-u_{2}\right\|_{L^{2}} .
\end{aligned}
$$

By the Strichartz estimate, letting $T$ smaller if necessary, we hence obtain

$$
d\left(Q\left[u_{1}\right], Q\left[u_{2}\right]\right) \leqslant \frac{1}{2} d\left(u_{1}, u_{2}\right)
$$

for any $u_{1}, u_{2} \in \mathcal{H}_{T, M}^{1,2}$.

A similar result as Lemma 3.2 holds since

$$
\left|\frac{d}{d t}\|\log \langle x\rangle u(t)\|_{L^{2}}^{2}\right|=\left|\operatorname{Im} \int \frac{2 x \log \langle x\rangle}{1+x^{2}} \cdot \nabla u(t) \overline{u(t)} d x\right| \leqslant C\|\nabla u(t)\|_{L^{2}} .
$$

Now, we have a priori bound of $\|\nabla u(t)\|_{L^{2}}$ as in the case $2 \leqslant p<3$ of Theorem 1.4, which proves the global well-posedness.

Acknowledgments. The author expresses his deep gratitude to Professor Patric Gérard for fruitful discussions. Deep appreciation goes to Professor Hideo Kubo for his valuable advice and constant encouragement. This research is supported by Grant-in-Aid for JSPS Fellows.

\section{REFERENCES}

1. A. Arnold and F. Nier, The two-dimensional Wigner-Poisson problem for an electron gas in the charge neutral case, Math. Methods Appl. Sci. 14 (1991), no. 9, 595-613.

2. R. Carles, Remarks on nonlinear Schrödinger equations with harmonic potential, Ann. Henri Poincaré 3 (2002), no. 4, 757-772.

3. _ Nonlinear Schrödinger equations with repulsive harmonic potential and applications, SIAM J. Math. Anal. 35 (2003), no. 4, 823-843 (electronic).

4. __ Global existence results for nonlinear Schrödinger equations with quadratic potentials, Discrete Contin. Dyn. Syst. 13 (2005), no. 2, 385-398.

5. R. Carles, N. J. Mauser, and H. P. Stimming, (semi)classical limit of the Hartree equation with harmonic potential, SIAM J. Appl. Math 66 (2005), no. 1, 29-56.

6. T. Cazenave, Semilinear Schrödinger equations, Courant Lecture Notes in Mathematics, vol. 10, New York University Courant Institute of Mathematical Sciences, New York, 2003.

7. M. De Leo and D. Rial, Well posedness and smoothing effect of Schrödinger-Poisson equation, J. Math. Phys. 48 (2007), no. 9, 093509, 15.

8. D. Fujiwara, Remarks on convergence of the Feynman path integrals, Duke Math. J. 47 (1980), no. 3, 559-600.

9. R. T. Glassey, On the blowing up of solutions to the Cauchy problem for nonlinear Schrödinger equations, J. Math. Phys. 18 (1977), no. 9, 1794-1797. 
10. R. Killip, M. Visan, and X. Zhang, Energy-critical NLS with quadratic potentials, Comm. Partial Differential Equations 34 (2009), no. 12, 1531-1565.

11. S. Masaki, Local existence and WKB approximation of solutions to SchrödingerPoisson system in the two-dimensional whole space, archived as arXiv:0912.1388, 2009.

12. Y.-G. Oh, Cauchy problem and Ehrenfest's law of nonlinear Schrödinger equations with potentials, J. Differential Equations 81 (1989), no. 2, 255-274.

13. M. Reed and B. Simon, Methods of modern mathematical physics. II. Fourier analysis, self-adjointness, Academic Press [Harcourt Brace Jovanovich Publishers], New York, 1975.

14. H. Steinrück, The one-dimensional Wigner-Poisson problem and its relation to the Schrödinger-Poisson problem, SIAM J. Math. Anal. 22 (1991), no. 4, 957-972.

15. H. P. Stimming, The IVP for the Schrödinger-Poisson-X $\alpha$ equation in one dimension, Math. Models Methods Appl. Sci. 15 (2005), no. 8, 1169-1180.

16. J. Stubbe, Bound state for two-dimensional Schrödinger-Newton equations, archived as arXiv:0807.4059, 2008.

17. K. Yajima, Smoothness and non-smoothness of the fundamental solution of time dependent Schrödinger equations, Comm. Math. Phys. 181 (1996), no. 3, 605-629.

18. P. Zhang, Wigner measure and the semiclassical limit of Schrödinger-Poisson equations, SIAM J. Math. Anal. 34 (2002), no. 3, 700-718 (electronic).

19. X. Zhang, Global wellposedness and scattering for $3 D$ energy critical Schrödinger equation with repulsive potential and radial data, Forum Math. 19 (2007), no. 4, 633-675.

Division of Mathematics, Graduate School of Information Sciences, ToHOKU UNIVERSity, SENDAi 980-8579, JAPAN

E-mail address: masaki@ims.is.tohoku.ac.jp 